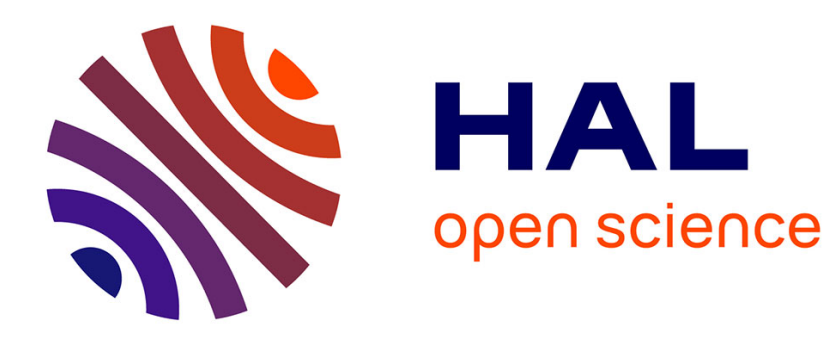

\title{
The entropy of a network of rod molecules
}

François Boué, S.F. Edwards, T.A. Vilgis

\section{To cite this version:}

François Boué, S.F. Edwards, T.A. Vilgis. The entropy of a network of rod molecules. Journal de Physique, 1988, 49 (10), pp.1635-1645. 10.1051/jphys:0198800490100163500 . jpa-00210844

\section{HAL Id: jpa-00210844 https://hal.science/jpa-00210844}

Submitted on 1 Jan 1988

HAL is a multi-disciplinary open access archive for the deposit and dissemination of scientific research documents, whether they are published or not. The documents may come from teaching and research institutions in France or abroad, or from public or private research centers.
L'archive ouverte pluridisciplinaire HAL, est destinée au dépôt et à la diffusion de documents scientifiques de niveau recherche, publiés ou non, émanant des établissements d'enseignement et de recherche français ou étrangers, des laboratoires publics ou privés. 
Classification

Physics Abstracts

$05.20-03.70-81.20$

\title{
The entropy of a network of rod molecules
}

\author{
F. Boué $\left({ }^{1}\right)$, S. F. Edwards $\left({ }^{2}\right)$ and T. A. Vilgis $\left({ }^{3}\right)$ \\ (1) Laboratoire Léon-Brillouin, CEN Saclay, F-91191 Gif sur Yvette, Cedex, France \\ ( $\left.{ }^{2}\right)$ Cavendish Laboratory, Madingley Road, Cambridge CB3 OHE, G.B. \\ (3) Max-Planck-Institut für Polymerforschung, PO-Box 3148, D-6500 Mainz, F.R.G.
}

(Reçu le 11 décembre 1987, accepté sous forme définitive le 20 mai 1988)

\begin{abstract}
Résumé. - Quoique les réseaux de polymères soient habituellement étudiés dans le cas totalement flexible, des réseaux sont maintenant synthétisés à partir de polymères de cristaux liquides dans lesquels le seul degré de liberté correspond à la rotation aux points de jonction. Ce sont les exemples les plus simples de systèmes avec éléments rigides ou presque rigides; ils présentent également un intérêt supplémentaire à la lumière des expériences récentes de diffusion de neutrons sur des réseaux habituels. Dans cet article, nous étudions des réseaux de bâtonnets rigides d'abord comme une extension de réseaux souples, ensuite par des méthodes nouvelles inventées pour ce problème. Les résultats montrent que, pour de petites déformations, l'énergie libre est très semblable à la loi en $\sum \lambda_{i}^{2}$ des réseaux habituels mais que, pour de grandes déformations, la variation est exponentielle en $\lambda$. Nous étudions aussi les enchevêtrements et nous prédisons une singularité en $\log \{1-\xi / a(\lambda-1)\}$ où $a$ est l'espacement entre les bâtonnets reliés à la concentration et $\xi$ est la longueur des bâtonnets.
\end{abstract}

\begin{abstract}
Although polymer networks are usually studied in the fully flexible case, there are many networks now being synthesized from liquid crystal polymers, where the only freedom lies in the hinging at the junction points. These provide the simplest examples of systems with rigid or nearly rigid members, also of interest in a view of recent neutron scattering experiments from conventional networks. In this paper we study rigid rod networks firstly as an extension of flexible networks, and secondly by new methods invented for this problem. The results show that for small deformations the free energy is very similar to usual $\sum \lambda_{i}^{2}$ law for conventional networks, but for large deformations the variation is exponential in $\lambda$. Entanglements are also studied and a singularity which behaves like $\log \{1-\xi / a(\lambda-1)\}$, where $a$ is the spacing of the rods related to the concentration and $\xi$ the rod length, is predicted from the free energy.
\end{abstract}

\section{Introduction.}

There are many reasons for studying the entropy of a rod network. The first is that they are tempted to be synthesized by Aharony [1] and Wegner [2]. It is now possible to have a network, whose basic ingredient is a liquid crystal polymer, with a freely or near freely hinged crosslink, of the type as given in the following figure 1 .

Maxwell [3] long ago showed that the rod network of this type is not rigid unless six or more members form a crosslink, hence the network has an entropy. The simplest theory of conventional rubbers, consisting of Gaussian chains (i.e. fully flexible polymers chains), is that of Wall [4] in which the crosslinks are assumed to be fixed in space as far as Brownian

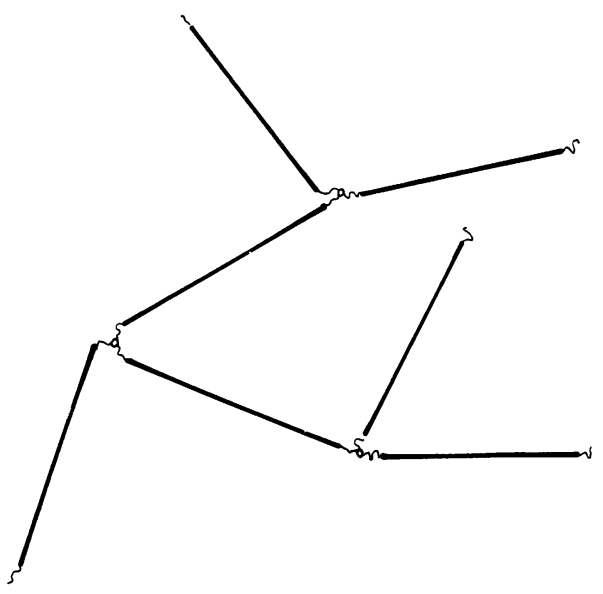

Fig. 1. - A model of a rod network with flexible hinges. 
motion is concerned. Further they are assumed to transform affinely during macroscopic deformation. Thus the Wall-Flory theories have an entropy of the rubber residing in the chains and not in the crosslinks. This underestimates the entropy of the network by a factor of 2 . In more general theories, such as in that of James and Guth [5] half of the entropy can be ascribed to the motion of the crosslinks. We make this point to show that there is a large amount of entropy in the rod network, even though at the first sight it would appear to contain $\ell / \xi$ fewer degrees of freedom compared to the fully flexible case. $\ell$ is the Kuhn step length of a corresponding polymer and $\xi$ the length of the rod.

The problem may be significant elsewhere. Rods or rather stiff molecules are often found from biological origin, e.g. collagen, carrageenan and many various other polysaccarides. This crosslinkage is however very complex, and their chemistry and chemical physics may be obscured by many simultaneous effects, so that their proportion of the free energy, which is enthalpic and entropic is not known. Nevertheless these substances must be more complicated than in the present study.

Finally we mention the actual origin of this paper which are surprising neutron scattering results of Bastide and Boué $[6,7]$, who do not find the results as expected from the classical theories, as for example given by Warner and Edwards [8] or Vilgis and Boué [9]. They find, after letting the permanent crosslinked system relax a long time, that the crosslink positions seem to keep their mutual distances constant and do not transform affinely. Instead they appear to move rather like the pins of a pantograph. The network of rods certainly does keep the crosslink positions at constant distances, and so, although we are not putting the present paper forward as a new theory of rubber elasticity, we are faced with the problem that experiments; although at short times Stein [10] obtains good agreement with the classical affine deformation model. Therefore the present calculation may serve as a useful ingredient as some comprehensive future treatment of this problem.

The plan of the paper is to do our best with the conventional theory in section 2 , and then apply some custom built new methods, introduced in the preceding paper before this one (hereafter refered as I) in section 3, finally we incorporate as best we may at present the problem of entanglements in section 4 .

\section{An extension of the current theory for rods (« first quantization » approach).}

The most conventional process for crosslinking rubbers leads to the functionality of four, as shown in figure 2 .

If we are concerned with very long chains with

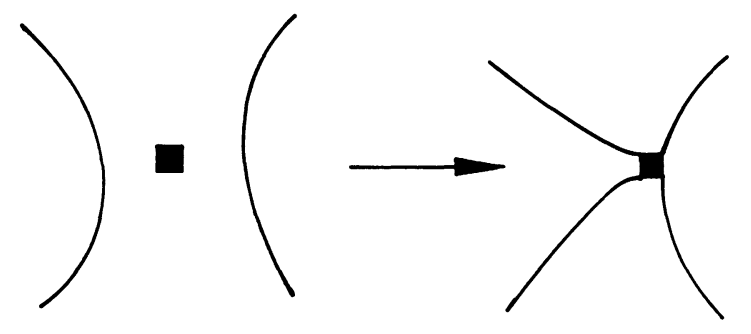

Fig. 2. - Conventional crosslinking of two objects, for example polymer chains leads to a four-functional links.

many crosslinks along their length, we can consider the rubber to consist of one huge molecule, self crosslinked, occupying the volume of the material with approximately uniform density. If these crosslink are at the arc points $s_{i}$ of the (giant) chain $\mathbf{R}(s)$, the problem is to evaluate the number of configurations of $\mathbf{R}(s)$ assuming it to be completely flexible apart from the crosslinks $\mathbf{R}\left(s_{i}\right)=\mathbf{R}\left(s_{j}\right)$ and the space filling condition. Then suppose the system to be deformed by a matrix $(\boldsymbol{\lambda})_{i j}=\lambda_{i} \delta_{i j}$, i.e. the principal strains are $\lambda_{i}-1$, and calculate the new entropy in this deformed state, but with the same crosslink positions.

The entropy experimentally observed in the undeformed state is given by a very general expression [12]

$$
S_{\exp }=\int S([\mathbf{s}]) P([\mathbf{s}]) \mathrm{d} \mathbf{s}
$$

where $\mathbf{s}$ is the matrix of intersections, i.e. if $s_{i}$ meets $s_{j}$ we may label them $s_{i}^{j}$, $s_{j}^{i}$ to form the crosslink. $P([\mathbf{s}])$ is the probability to place the crosslinks at all $\left\{s_{i}^{i}\right\}$ and $\left\{s_{j}^{i}\right\}$ along the contour. $S([\mathrm{~s}])$ is given by the number of configurations $\Omega([\mathbf{s}])$ with s via Boltzmann's famous equation

$$
S([\mathbf{s}])=k_{\mathrm{B}} \log \Omega([\mathbf{s}]) .
$$

The simplest hypothesis for the probability $P([\mathbf{s}])$ assumes

$$
P([\mathbf{s}])=\Omega([\mathbf{s}]) / \bar{\Omega}
$$

with $\bar{\Omega}=\sum_{[\mathrm{s}]} \Omega([\mathrm{s}])$. Thus we find

$$
S_{\exp }=\frac{\int \mathrm{d} \mathbf{s} \Omega([\mathbf{s}]) \log \Omega([\mathbf{s}])}{\int \mathrm{d} \mathbf{s}^{\prime} \Omega\left(\left[\mathbf{s}^{\prime}\right]\right)}
$$

which is the usual formula for quenched averages [11-14]. In the following we will consider $\Omega$ to be normalized and the integral in the denominator is equal to 1 .

The same equation holds also for the calculation 
of the free energy in the deformed state where $S_{\text {exp }}(\boldsymbol{\lambda})$ can be written as

$$
S_{\exp }(\boldsymbol{\lambda})=\int \mathrm{ds} \Omega([\mathbf{s}]) \log \Omega([\mathbf{s}], \boldsymbol{\lambda}) .
$$

Hence $\Omega$ has to be initial state of the distribution and does not contain the deformation tensor $\boldsymbol{\lambda}$, but the log-term does. This becomes clear if we think of calculating the free energy of deformation a particular network with configuration $\mathbf{s}$ at formation conditions and then averaging over all possible values of $\mathbf{s}$ with the appropriate weight. The replica method [11-13] is available to handle the averaging of the logarithmic term, in which $\log \Omega$ is replaced by $\left(\mathrm{d} \Omega^{n} / \mathrm{d} n\right)_{n=0}$. Taking the average of $\Omega^{n}$ is much simpler, and the final answer for the free energy is given by the coefficient in an $n$-expansion of

$$
S_{n}=\int \mathrm{d} \mathbf{s} \Omega([\mathbf{s}]) \Omega^{n}([\mathbf{s}], \boldsymbol{\lambda}) .
$$

For flexible polymers it can be shown easily that $\Omega^{n}([\mathbf{s}], \boldsymbol{\lambda})$, or more generally, the partition function in the deformed state has the version of the replicated Edwards Hamiltonian written in the Wiener integral form $[12,14,15]$

$$
\begin{aligned}
\left\langle Z^{n}\right\rangle= & \int \frac{\mathrm{d} \mu M !}{2 \pi i \mu^{M+1}} \int \prod_{\alpha=0}^{n} \delta \mathbf{R}^{(\alpha)} \times \\
& \times \exp \left(-\sum_{\alpha=0}^{n} \frac{3}{2 b} \int_{0}^{L} \mathbf{R}^{\prime(\alpha)^{2}} \mathrm{~d} s+\right. \\
& \left.+\mu \int_{0}^{L} \int_{0}^{L} \prod_{\alpha=0}^{n} \mathrm{~d} s \mathrm{~d} s^{\prime} \delta\left\{\mathbf{R}^{(\alpha)}(s)-{ }^{(\alpha)}\left(s^{\prime}\right)\right\}\right)
\end{aligned}
$$

where the $\mathbf{R}^{(0)}$ corresponds to the undeformed state and all variables $\mathbf{R}^{(\alpha)}, \alpha=1 \ldots n$ are « polymers » in the rubber deformed by a tensor $\boldsymbol{\lambda}$, and $\mu$ is the fugacity for $M$, i.e. the number of crosslinks inserted. $b$ is the Kuhn length. An evaluation of this integral and expanding in $n$ « readily " gives the James and Guth result [5], i.e.

$$
S=1 / 2 M\left(\lambda_{1}^{2}+\lambda_{2}^{2}+\lambda_{3}^{2}\right)
$$

where $M$ is the number of crosslinks. For details see $[11,12,14]$.

The great simplification in (2.6) is the Gaussian structure of the integrals which enables one to guess a trial approximation which says that if we assume that each of the $\mathbf{R}^{(\alpha)}$ is coupled by an harmonic constraint to the affinely deformed $\mathbf{R}^{(0)}$, so that a managable calculation is ensured.

In the present problem we only carry the crosslink points since for an intermediate point within the rod no deformation occurs. Thus taking the network to be a huge polymer of straight segments, each of

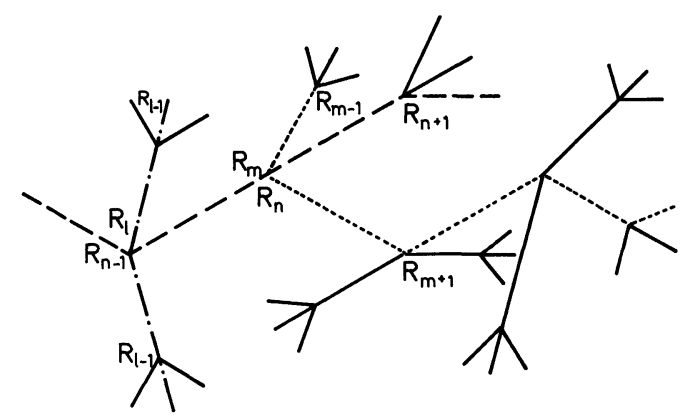

Fig. 3. - A rod tree network with three-fold crosslinks. A path with links at labels $m-1, m, m+1$ is crosslinked with the path $n-1, n, n+1$ at $\mathbf{R}_{n}=\mathbf{R}_{m}$. The path with the 1 labes is crosslinked with the path of $n$ labels at $\mathbf{R}_{1}=\mathbf{R}_{n-1}$, and so on.

length $\xi$, the whole network can be considered as a set of points $\mathbf{R}_{0}, \mathbf{R}_{1}, \mathbf{R}_{2}, \ldots$, where there is a crosslinkage of the form $\mathbf{R}_{n}=\mathbf{R}_{m}$ (see Fig. 3).

The network is all connected but labelling suggests particular strands crosslinking as in the hatching of the diagram. Thus if

$A\left(\mathbf{R}_{i}, \mathbf{R}_{i+1}\right)=1 /\left(4 \pi \xi^{2}\right) \delta\left(\left|\mathbf{R}_{i}-\mathbf{R}_{i+1}\right|-\xi\right)$

describes the elements of the network with the appropriate crosslink positions the new structure of the network is given by

$$
\prod_{k=0}^{N} A\left(\mathbf{R}_{k}, \mathbf{R}_{k+1}\right) \prod_{i, j}^{M} \delta\left(\mathbf{R}_{i}-\mathbf{R}_{j}\right)
$$

where $\prod A$ describes the giant network, and the product $(i, j)$ is over those places which are crosslinked. $N$ is the number of rods and $M$ the number of crosslinks. We can now introduce a fugacity $\mu$ (as given in detail in references $[11,12$, 14] and in I) to replace the product over the crosslink positions. The partition function for the undeformed state can the be written as

$$
\begin{gathered}
\langle Z\rangle=\oint \frac{\mathrm{d} \mu M !}{2 \pi i \mu^{M+1}} \int \prod_{m} \delta \mathbf{R}_{m} A\left(\mathbf{R}_{m}, \mathbf{R}_{m+1}\right) \times \\
\times \exp \left(\mu \sum_{i, j} \delta\left(\mathbf{R}_{i}-\mathbf{R}_{j}\right)\right) .
\end{gathered}
$$

This has to be extended to a replicated form and the precise form is given by

$$
\begin{aligned}
\left\langle Z^{n}\right\rangle= & \oint \frac{\mathrm{d} \mu M !}{2 \pi i \mu^{M+1}} \times \\
& \times \int \prod_{\alpha=0}^{n} \prod_{m} \delta \mathbf{R}_{m}^{(\alpha)} A\left(\mathbf{R}_{m}^{(\alpha)}, \mathbf{R}_{m+1}^{(\alpha)}\right) \times \\
& \times \exp \left(\mu \sum_{i, j} \sum_{\alpha=0}^{n} \delta\left\{\mathbf{R}_{i}^{(\alpha)}-\mathbf{R}_{j}^{(\alpha)}\right\}\right) .
\end{aligned}
$$


This partition function is not easy to evaluate, but it can be done in a two stage approximation. Again one might try an harmonic constraint in modelling the effect of the crosslinks. The harmonic trial potential has to give the «best fit » to the crosslinks and the function A. "Best fit » means a variational principle of the Feynman type [16], and for Gaussian chains an harmonic trial potential $U \sim q_{0}^{2} \int \mathbf{R}^{2}(s)$ leads to minimizing the free energy. This process determines $q_{0}$. In the case of flexible chains $q_{0}$ turns out to be $q_{0}=1 /\left\langle R_{0}^{2}\right\rangle$, i.e. the inverse of the mean square of the radius of gyration of a rubber strand between two crosslinks.

For rods this variational method turns out to be akin to a cluster expansion in usual statistical mechanics, now in $3 n+3$ dimensional space. We leave an outline of the analysis to the appendix and quote only the result here. The free energy is given by

$$
\begin{aligned}
F / k_{\mathrm{B}} T=\frac{1}{4} \ell^{2} \sigma \alpha\left(q_{0}\right) / & \left\{1+q_{0}^{2}\left(\ell^{2} \sigma\right)^{2} / 36\right\}^{1 / 2}-M / 2 \log \left\{\alpha\left(q_{0}\right) / \pi\right\}^{3 / 2}+M / 2 \sum_{i} \lambda_{i}^{2}+ \\
+ & M\left\{\alpha\left(q_{0}\right) / \pi\right\}^{3 / 2}\left\{\int \mathrm{d}^{3} \varepsilon \exp \left\{-\frac{\alpha}{2}\left(\varepsilon^{2}-\xi^{2}\right)\right\} \frac{\sinh 2|\boldsymbol{\varepsilon}| \xi}{2|\varepsilon| \xi}\right. \\
\times & {\left.\left[\sum_{i} \lambda_{i}^{2}-\frac{2}{\alpha\left(q_{0}\right)} \sum_{i} \lambda_{i}^{2} \varepsilon_{i}^{2}+\log \left\{\exp \left(-\alpha\left(q_{0}\right) \xi^{2} / 2\right)\right\} \frac{\sinh 2|\varepsilon \boldsymbol{\lambda}| \xi}{2|\varepsilon \boldsymbol{\lambda}| \xi}\right]\right\} }
\end{aligned}
$$

where

$$
\alpha\left(q_{0}\right)=\frac{1}{2} q_{0}\left\{1+\left(q_{0} \ell^{2} \sigma\right)^{2} / 36\right\}^{1 / 2} .
$$

$\varepsilon$ is a unit vector and $\mathrm{d} \varepsilon$ is an integration over a sphere with radius 1 . The auxillary variables $\sigma$ and $\ell$ are defined as follows. In the variational calculation it turns out to be useful to devide the rod in $\sigma$ parts each of length $\ell$. If the rod is turned to a flexible polymer, $\ell$ plays the same role as the Kuhn length and $\sigma$ is then the number of statistical segments. This enables to define an equivalent Gaussian limit of flexible networks is given within this approximation by $q_{0} \rightarrow 0$ so that quadratic orders in $\alpha$ can be neglected, i.e. $\alpha=q_{0} / 2$. To make the free energy stationary we have to minimize it with respect to the parameter $q_{0}$. For this artificial Gaussian limit the value $q_{0}=6 M / \ell^{2} \sigma$ is recovered [21]. Hence $\ell^{2} \sigma=\xi^{2}$ (see appendix A). This corresponds to an equivalent Gaussian network where all rods of length $\xi$ have been replaced by a chain with contour length $\sigma \ell$, but note that this is only possible in the weak crosslinking limit, i.e. $q_{0}$ small.

In the general case the minimization process becomes hard to do. It is analytically only possible in crude approximations.

Let us discuss the deformation dependence of the free energy qualitatively, without calculating the integrals over the sphere. The most surprising result is that for small deformations the elastic behaviour is just the same as for a network synthesized from flexible polymers, and the modulus is similar to that of the James and Guth model. The high deformation limit is different from that of conventional networks and we find apart from the usual $\lambda^{2}$ terms (all collected in a $F_{0}$ ) an exponential term of the form

$$
F \approx F_{0}+\beta \exp \left\{\gamma\left(\lambda_{i} \xi\right)^{2}\right\} \text {. }
$$

Thus we find a severe increase of the free energy at large extensions. $\beta$ and $\gamma$ are constants.

Consider now the limit of strong localization, i.e. $q_{0} \rightarrow \infty$. Then the result for the localization parameter is given by

$$
q_{0}=12 M / \xi^{2},
$$

which is a value double of that of the Gaussian limit and fluctuations appear to be more surpressed. The weak localization limit for flexible polymers is extensively discussed within this framework in Deam and Edwards [12] or Warner and Edwards [9]. For rods this is not possible in a simple manner.

This approach is very crude and the main criticism is that the Gaussian fitting might be not very convincing. There are clearly two severe drawbacks in this approach. Firstly it is restricted to four functional crosslinks, which although correctly mirrors the crosslinking process in vulcanization. This method is inadequate for the more general case of arbitrary crosslink functionality. Secondly the individual segments are of random length in the Gaussian model (see appendix).

One would really like a method which can handle segments of arbitrary specification and arbitrary functionality. The preceding paper I offers a field theory which does both of these things and we apply it in the next section.

\section{A field theory or arbitrary networks (the « second quantization approach $»$ ).}

The idea is very simple : a segment of type (a) with an end to end probability of $g^{a}\left(\mathbf{R}-\mathbf{R}^{\prime}\right)$ is represented by

$$
\int \mathrm{d}^{3} r \int \mathrm{d}^{3} r^{\prime} \Phi^{a}(\mathbf{r}) g^{a}\left(\mathbf{r}-\mathbf{r}^{\prime}\right) \Phi^{a}\left(\mathbf{r}^{\prime}\right)
$$


where the operator $\Phi^{a}(\mathbf{R})$ creates an end at $\mathbf{R}$. Crosslinks are represented by annihilation operator $\Phi^{a^{*}}$, so that

$$
\int \mathrm{d}^{3} r\left\{\Phi^{a^{*}}(r)\right\}^{m}
$$

will join up $m$ of the $\Phi^{a}$ s, i.e. the functionality is $m$. As explained in the preceeding paper $I$ the generality is that one can think of all sorts of combinations, like

$$
\int \mathrm{d}^{3} r \Phi^{a^{*}}(r) \Phi^{b^{*}}(r) \Phi^{c^{*}}(r) \Phi^{d^{*}}(r)
$$

where the $a, b$, etc. denote all different species. The mechanics of this formalism is already explained in I.

This is, of course, almost conventional field theory, but in order to take quenched averages into account it has to be extended to the replica method. Therefore the field variables $\Phi(\mathbf{r})$ have to be extended to $\Phi\left(\mathbf{r}^{(0)}, \mathbf{r}^{(1)}, \mathbf{r}^{(2)}, \ldots, \mathbf{r}^{(n)}\right)$, where $(\alpha)$ are the replicas again, and $n$ the number of replicas. One of the most remarkable features of I was that the simplest realization of the field equations permit a return to the replica space back to explicit formulae. Thus, according to the simplified version (see last paragraph in I), that by disregarding the loops in the network, the problem is reduced to solving the non linear integral equation for the mean-field values of $\bar{\Phi}$,

$$
\begin{aligned}
\int \mathrm{d}^{3} r^{\prime} g\left(\mathbf{r}-\mathbf{r}^{\prime}\right) \bar{\Phi}\left(\mathbf{r}^{\prime}\right) & = \\
& =\mathcal{C}(\mu, \nu)[\bar{\Phi}(\mathbf{r})]^{\frac{1}{m-1}}
\end{aligned}
$$

or the equivalent equation in $\bar{\Phi} *$

$$
\bar{\Phi} *(\mathbf{r})=\tilde{\mathcal{C}}(\mu, \nu) \int \mathrm{d}^{3} r^{\prime} g\left(\mathbf{r}-\mathbf{r}^{\prime}\right)\left\{\bar{\Phi}^{*}\left(\mathbf{r}^{\prime}\right)\right\}^{m-1} .
$$

The constants $\mathcal{C}(\mu, \nu)$ and $\tilde{\mathcal{C}}(\mu, \nu)$ depend later on the steepest decent values of the fugacities, and can be simply calculated. Since these functions are not important for our further considerations we do not write them out.

According to the results derived in paper I we have to evaluate

$$
\begin{aligned}
\mu \int \mathrm{d}^{3} R \int \mathrm{d}^{3} R^{\prime}\left[\int \mathrm{d}^{3} r \int \mathrm{d}^{3} r^{\prime} \bar{\Phi}(\mathbf{r}-\mathbf{R})\right. & g\left(\mathbf{r}-\mathbf{r}^{\prime}\right) \bar{\Phi}\left(\mathbf{r}^{\prime}-\mathbf{R}^{\prime}\right) \times \\
& \left.\times \log \left\{\int \mathrm{d}^{3} r \int \mathrm{d}^{3} r^{\prime} \overline{\boldsymbol{\Phi}}(\mathbf{r}-\boldsymbol{\lambda \mathbf { R }}) g\left(\mathbf{r}-\mathbf{r}^{\prime}\right) \bar{\Phi}\left(\mathbf{r}^{\prime}-\boldsymbol{\lambda} \mathbf{R}^{\prime}\right)\right\}\right]
\end{aligned}
$$

and

$$
\nu \int d^{3} R\left[\int d^{3} r\left\{\bar{\Phi}^{*}(\mathbf{r}-\mathbf{R})\right\}^{m} \log \left\{\int d^{3} r\left\{\bar{\Phi}^{*}(\mathbf{r}-\boldsymbol{\lambda} \mathbf{R})\right\}^{m}\right\}\right]
$$

where $\mu$ and $\nu$ are the appropriate fugacities.

For 3 fold functionality and in the case of rods we have

$$
\bar{\Phi} *(r)=\tilde{\mathcal{C}}(\nu, \mu) \int \mathrm{d}^{3} r^{\prime} \delta\left(\left|\mathbf{r}-\mathbf{r}^{\prime}\right|-\xi\right)\left\{\overline{\boldsymbol{\Phi}}^{*}\left(\mathbf{r}^{\prime}\right)\right\}^{2}
$$

where we have been now evaluating $\bar{\Phi}^{*}(r)$ instead of the $\bar{\Phi}$ 's (both are allowed of course). Equation (3.6) can be written as

$$
\bar{\Phi}^{*}(r)=\tilde{\mathbf{C}}^{\prime}(\nu, \mu) \int \mathrm{d} \boldsymbol{\varepsilon}\{\bar{\Phi} *(\mathbf{r}-\varepsilon \xi)\}^{2}
$$

where $d \varepsilon$ is the same unit sphere integration as in the preceding section. This equation has a ready physical meaning in that the freedom $\bar{\Phi}$ associated with two rods flows into a third.

Concerning the solution of the integral equation remember that for Gaussian chains the solution has to be Gaussian and one might guess this form.
Hence for 3 functional crosslinked flexible molecules we had (see I)

$$
\mathrm{e}^{-\alpha \mathbf{r}^{2}}=\mathcal{C}(\nu, \mu) \int \mathrm{d}^{3} r^{\prime} \mathrm{e}^{-\beta\left(\mathbf{r}-\mathbf{r}^{\prime}\right)^{2}-2 \alpha \mathbf{r}^{2}}
$$

giving the solution $\alpha=\beta / 2$. There is no such a simple solution for the case of crosslinked rods and one enters the usual difficulties for non linear integral equations. We have to rely on approximations, so let us discuss the properties of the solution now.

Near $r=0$ equations $(3.6,3.7)$ can be forced crudely to a Gaussian. But if we follow this lines we 
would have the same calculation as in the flexible case but with $\beta=1 / 2 \xi^{2}$. This type of calculation is very similar to that in section 2 . Note that this replaces the rod by an effective Gaussian of the form $\exp \left\{-\left(\mathbf{r}-\mathbf{r}^{\prime}\right) / 2 \xi^{2}\right\}$, which is usually very bad, since it is equivalent to the rod length fluctuating.

An improvement is to notice the Fourier transform of the structure function of the rod, i.e.

$$
\mathcal{F} \mathcal{G}\left[\frac{1}{4 \pi \xi^{2}} \delta\left(\left|\mathbf{r}-\mathbf{r}^{\prime}\right|-\xi\right)\right] \sim \frac{\sin (k \xi)}{k \xi}
$$

which may be reasonable approximated by $1 /\left\{1+(k \xi)^{2} / 3\right\}$ inasmuch as it is correct near $k=0$, and small for large $k$. One can then transform the integral equation into a differential equation

$$
\Psi-\left(\xi^{2} / 3\right) \nabla^{2} \Psi=\mathcal{C}(\nu, \mu) \Psi^{2}
$$

where $\Psi=\Phi^{*}$, or more generally for arbitrary functionalities we have

$$
\Psi-\left(\xi^{2} / 3\right) \nabla^{2} \Psi=\mathcal{C}(\nu, \mu) \Psi^{m-1} .
$$

A variational solution can now be tried, and remarked above, if a Gaussian is used, the coefficient will be the same as for flexible polymers (but with a different meaning). Hence the case $m=2$ is a chain of rigid rods and is trivial, since it gives a Gaussian chain for a sufficient large number of rods.

The original equation allows to study large deformations, for this we solve the equations for large arguments. In this case the integral has its value concentrated at the point $\varepsilon=\mathbf{r} /|\mathbf{r}|$, since the function $\bar{\Phi} *(\mathbf{r})$ decreases with $\mathbf{r}$ and $\mathbf{r}-\xi \varepsilon$ is the point in the integration where $\Phi^{*}$ is largest. Asymptotically this means that $\log \Phi^{*}$ behaves exponentially, and we have the following approximate equations

$$
\bar{\Phi}^{*}(|\mathbf{r}|)=\mathcal{C}(\nu, \mu) \bar{\Phi}^{* 2}(|\mathbf{r}|-\xi)
$$

and

$$
\bar{\Phi} *(|\mathbf{r}|)=A \exp \left[-\alpha \mathrm{e}^{\beta r / \xi}\right]
$$

for the left hand side, and

$$
\mathcal{C} \bar{\Phi} *(|\mathbf{r}|)=(A \mathcal{C})^{2} \exp \left[-2 \alpha \mathrm{e}^{\beta r / \xi-\beta}\right]
$$

for the right hand side. Hence $\beta=\log 2$ for a solution and the remaining integration over the unit vector $\varepsilon$ just leaves a logarithmic correction.

Returning to equation (3.7) we see that the integral has most of its value near the various origin of integration, but as suggested by equation (3.5) $\lambda \mathbf{R}$ is far from its origin when the elements of the deformation tensor are large. Consider an uniaxial deformation $R \rightarrow \lambda R$ we have

$$
\begin{aligned}
& \iint \Phi g \Phi \equiv \\
& \equiv \iint \Phi^{* 2} g \Phi^{* 2} \underset{\lambda \rightarrow \infty}{\Longrightarrow} \int \mathrm{d}^{3} r \Phi^{* 2}(r-\lambda R) \times \\
& \quad \times g\left(R-R^{\prime}\right) \Phi^{* 2}\left(r-\lambda R^{\prime}\right) \cong \\
& \cong \exp \left[-\alpha \mathrm{e}^{\beta \lambda\left(R-R^{\prime}\right)}\right]
\end{aligned}
$$

to lowest order. $\alpha$ and $\beta$ are unidentified but appropriate constants as before. The free energy involves now averages as given in (3.5) and we find for the large deformation part asymptotically

$$
F=\text { const } \exp \left\{(\beta \lambda)^{2}\right\}
$$

as before in section 2. Crude as this evaluation is, it shows that for large extensions there is a fast increase in the free energy, essentially due to the inextensibility and the stiffness of the elements.

\section{Entanglements.}

The effect of entanglements in conventional rubbers have been studied to some detail by Edwards and Vilgis $[14,17]$ on the basis of sliplinks and tubes. It has been shown in these references that one might expect severe alterations from the classical phantom type theories, where the basic assumption was that the chains can pass through each other. Clearly, the preceeding sections consider " phantom rods", too, and we have to argue about entanglement constraints in the rod network also. Entanglements in isotropic melts or dense solutions of rods (above nematic transitions) have been considered by Doi and Edwards and the results are now collected in their book [18]. In essence it has been shown that these entanglement constraints are responsible for very slow dynamics, and are even responsible for a glass transition [19]. Thus we expect as in the case of flexible molecules similar additional complications.

The entanglement has to be considered in a more general way for the rod system, both in principle and in practise. In principle, because finite rods do not entangle, except on a temporary basis ; it is essential that they form part of the network. Thus for the method described in section 2 we could adopt the concept of the Gauss integral [20], but this is not sensible for the more general field theoretical analysis of section 3 . More convincing would be to say that the rods do not pass through each other during deformation, i.e. as soon as they touch, the entanglement constraint begins to work. Let us take

$$
\begin{aligned}
\int_{0}^{\xi} \mathrm{d} s \int_{0}^{\xi} \mathrm{d} s^{\prime} \delta\left\{(\xi-s) \mathbf{R}_{1}-s \mathbf{R}_{2}-\right. \\
\left.-\left(\xi-s^{\prime}\right) \mathbf{R}_{3}+s^{\prime} \mathbf{R}_{4}\right\} .
\end{aligned}
$$

This function vanishes unless the rod $\mathbf{R}_{1}, \mathbf{R}_{2}$ meets 
the $\operatorname{rod} \mathbf{R}_{3}, \mathbf{R}_{4}$. Suppose these rods are in a space deformed by a strain. Then the rods themselves are not deformed, but we can move their centres affinely and rotate them in accord with the strain tensor at the centre. If then the integral is then evaluated at a strain $\lambda$, the condition to be imposed is that the integral remains zero. In practice this is not an easy function to handle, but we would like to give some estimate of when entanglements matter and to this end give a crude model. The suggestion of Doi and Edwards [18] in their treatment of rod suspensions, suggest that the one can visualize each rod surrounded by a tube, formed by all other rods. It has been shown that, provided these neighbours are sufficiently numerous, a theory of viscoelasticity can be developed.

Let us suppose this idea can be adopted here. Then, in order to draw a diagram, mark the constraining rods by dots (i.e. rods perpendicular to the paper plane) and look at a piece of the network which is represented in figure 4 .

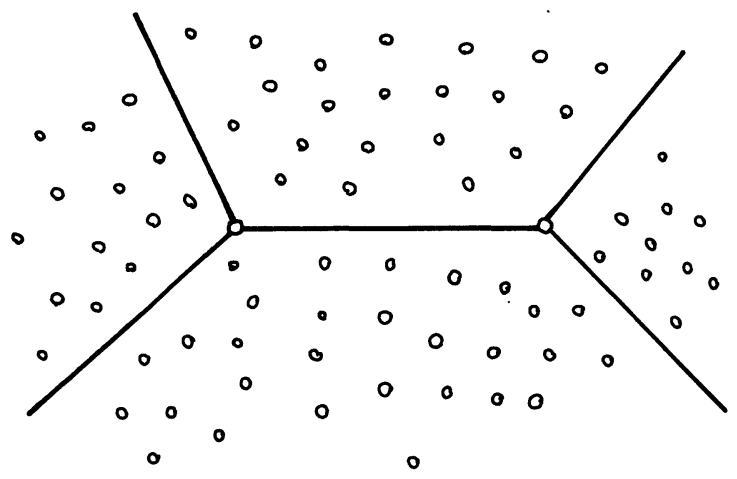

Fig. 4. - A dense network of crosslinked rods. The dots correspond to rods coming out of the paper plane in this two dimensional drawing. This is an entangled network, similar to the case of flexible chains [14].

The dots are symbols for rods coming out of the paper plane. This picture is a great simplification, but it enables to derive various sensible results [18, 19]. The dots an now replaced by a tube model as shown in figure 5.

The radius of the tube $a$ will be $(\rho \xi)^{-1 / 2}$, where $\rho$ is the number density of rods. Thus the tube radius scales like the rod concentration $c^{-1 / 2}$ in appropriate units.

Take the simplest geometry first (Fig. 6) and ignore all the «dotted » rods in figure 4.

Under deformation $\lambda$, the tube axis which starts at $2 a+\xi$ are moved to $\lambda(2 a+\xi)$. Thus the freedom of the rod is reduced from

$$
2 a=\xi-(\xi-2 a)
$$

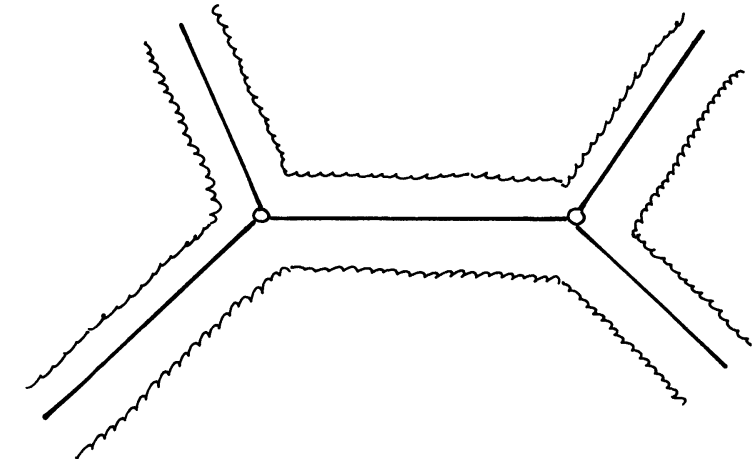

Fig. 5. - The same as in figure 4. The effect of neighbouring rods has been replaced by a tube.

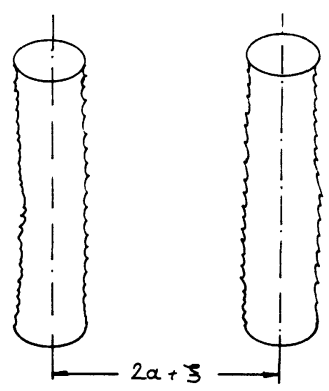

Fig. 6. - See text.

to the value

$$
\xi-\lambda(\xi-2 a)=\xi(1-\lambda)+2 a \lambda .
$$

We can therefore argue a change in entropy of $k_{\mathrm{B}} \log (2 a)$ to $k_{\mathrm{B}} \log (\xi(1-\lambda)+2 a \lambda)$. This has now the familiar crisis of the imperfect gas which takes the finite extension of the molecules into account. Thus one finds a maximum extension $\lambda_{\max }$ at

$$
\xi(1-\lambda)+2 a \lambda=0
$$

giving

$$
\lambda_{\max }=\xi /\{\xi-2 a\} .
$$

Thus by this crude argument, where all orientational correlations have been ignored, we argue that at a value of $\lambda$ of $\xi /\{\xi-2 a\}$ will lead to the network locking due to entanglements. It follows that one can ignore entanglements provided that $\xi<c^{-1 / 2}$, but if $\xi$ is of order of $c^{-1 / 2}$, they will play an important role in the modulus and maximum extensibility of the network. The freedom given in the previous section to the crosslinks to be of order $\xi$, we reach the non surprising result that the work of section 2 and 3 fits in the result obtained here.

\section{Conclusion.}

We find that it is possible in principle to write out a theory for the entropy of networks containing arbit- 
rary element, although the form of explicit solutions seem to be difficult, albeit explicit integral equations and the evaluation of difficult integrals. Two problems emerge from the analysis. Firstly that it is possible to take explicit detailed liquid crystal polymer networks and calculate the elastic properties and we hope to do this in detail in a later paper. Secondly that entropy is obviously only one part of a complete analysis and to this must be added in the energy arising from the bending energy, orientational energy, changes of the angles near the crosslinks which are seldom as simple as the freely hinged case, etc.

Nevertheless both papers show how one can extent field theory to networks giving now a unified picture to all the classical phantom theories, and how to extend the theory for arbitrary networks in one of the most general way. But it shows limitations as well. Dispite the combination of field theory and the replica approach for quenched variables which seems to be a very powerful method, limitations are given by the analysis, for example by the solution of non-linear integral equations.

\section{Acknowledgment.}

Helpful discussions on experimental problems with
Prof. G. Wegner (MPI Mainz) are gratefully acknowledged. We had also many useful conversation with Drs. R. C. Ball, J. Bastide, J. F. Joanny, and M. Warner.

\section{Appendix A.}

OUTLINE OF THE CALCULATION OF THE FIRST QUANTIZATION TYPE METHOD. - We wish to calculate the partition function of the type

$$
\begin{array}{r}
Z=\int \prod \mathrm{d}^{3} R_{m} \prod_{m}^{N} \delta\left(\left|\mathbf{R}_{m}-\mathbf{R}_{m+1}\right|-\xi\right) \times \\
\times\left[\sum_{i, j} \delta\left(\mathbf{R}_{i}-\mathbf{R}_{j}\right)\right]^{M}
\end{array}
$$

where we have omitted unimportant prefactors and normalization constants. In the following we go along the same lines as reported in detail by Deam and Edwards [12] and we will be brief here. The power can be exponentiated by employing a fugacity (or chemical potential) for the crosslinks giving (as given in detail in Refs $[12,14]$ and in I) the partition function for the undeformed state

$$
Z=\oint \frac{\mathrm{d} \mu M !}{2 \pi i \mu^{M+1}} \int \prod_{m} \mathrm{~d}^{3} R_{m} \prod_{m}^{N} \delta\left(\left|\mathbf{R}_{m}-\mathbf{R}_{m+1}\right|-\xi\right) \exp \left[\mu \sum_{i, j} \delta\left(\mathbf{R}_{i}-\mathbf{R}_{j}\right)\right]
$$

The replicated form is

$$
\begin{aligned}
\left\langle Z^{n}\right\rangle=\oint \frac{\mathrm{d} \mu M !}{2 \pi i \mu^{M+1}} \int \prod_{\alpha=0}^{n} \prod_{m} \delta \mathbf{R}_{m}^{(\alpha)} \prod_{\alpha=0}^{n} \prod_{m}^{N} \delta\left(\left|\mathbf{R}_{m}^{(\alpha)}-\mathbf{R}_{m+1}^{(\alpha)}\right|-\xi\right) \times & \\
& \times \exp \left[\mu \sum_{i, j} \sum_{\alpha=0}^{n} \delta\left\{\mathbf{R}_{i}^{(\alpha)}-\mathbf{R}_{j}^{(\alpha)}\right\}\right] .
\end{aligned}
$$

Let us write this more symbolically

$$
Z^{n}=\int \exp (\mu X+R)
$$

where $\mu X$ is the crosslink term and $R$ is the exponentiated form of the term coming from structure of the rods. As usual we simulate the crosslink term according to the variational principle by an harmonic potential, i.e.

$$
U=\frac{1}{6} q_{0}^{2} \ell \sum_{\alpha=1}^{n} \sum_{m}\left\{\mathbf{R}_{m}^{(\alpha)}-\lambda \mathbf{R}_{m}^{(0)}\right\}
$$

The meaning of $\ell$ will become clear later. One may think of dividing the rod in a number of « Kuhn steps » of length $\ell$ to get the equivalent flexible polymer, i.e. with an mean square end to end separation of $\xi^{2}$.
For the variational principle let us write the partition function as

$$
\int \mathrm{e}^{\mu X+R}=\int \mathrm{e}^{-W-U+W+U+\mu X+R}
$$

As a trial for $W$ we take an discrete «Wiener measure », i.e. the Gaussian

$$
W=\frac{3}{2 \ell \sigma} \sum_{\alpha=1}^{n} \sum_{m}\left\{\mathbf{R}_{m}^{(\alpha)}-\mathbf{R}_{m+1}^{(\alpha)}\right\}^{2}
$$

where $\sigma \ell \approx \xi^{2}$. The variational principle says now that (A.6) may be replaced by

$$
\left\langle Z^{n}\right\rangle=\int \mathrm{e}^{\mu X+R} \leqslant \int \mathrm{e}^{-W-U+\langle W+U+\mu X+R\rangle}
$$


where the average has to be taken over $\int \mathrm{e}^{-W-U}$. It is the latter averaging which relates the quantities $\sigma$ and $q_{0}$ to that of the initial partition function, to give the best fit.

One of the most severe problem in the calculation is how to handle the $\mathrm{e}^{R}$ term. Let us therefore write

$$
\begin{aligned}
R+W & =\log \left\{\prod_{\alpha=0}^{n} \prod_{m}^{N} \delta\left(\left|\mathbf{R}_{m}^{(\alpha)}-\mathbf{R}_{m+1}^{(\alpha)}\right|-\xi\right)\right\}+W \\
& =\log \left\{\prod_{\alpha=0}^{n} \prod_{m}^{N} \delta\left(\left|\mathbf{R}_{m}^{(\alpha)}-\mathbf{R}_{m+1}^{(\alpha)}\right|-\xi\right) \exp (W)\right\} \\
& =\log \left\{\prod_{\alpha=0}^{n} \prod_{m}^{N} \delta\left(\left|\mathbf{R}_{m}^{(\alpha)}-\mathbf{R}_{m+1}^{(\alpha)}\right|-\xi\right) \exp \left(\frac{3}{2 \ell \sigma} \sum_{\alpha=1}^{n} \sum_{m}\left\{\mathbf{R}_{m}^{(\alpha)}-\mathbf{R}_{m+1}^{(\alpha)}\right\}^{2}\right)\right\}
\end{aligned}
$$

so that they go well together. Next the $\delta$-function should be exponentiated, and again we employ a crude trick. Symbolically we write $\delta \mathrm{e}^{\sum W}=$ $1+\sum Q$, and we try a cluster expansion

$$
\log \prod(1+Q) \approx \sum Q
$$

Thus we have « sandwiched » the $\delta$-function between two exponentials of the Wiener type, but with opposite sign.

All averages can now be calculated (for some advice see $[8,12]$ if one recognizes the transformation matrix $T$ of the replicated coordinates
$\left\{\mathbf{R}^{(\alpha)}\right\}=\mathbf{T}^{(\alpha, \beta)}\left\{\mathbf{X}^{(\beta)}\right\}$, i.e. one can define « centre of mass " coordinate and the rotated coordinates in a $3 n+3$ dimensional space by the relations

$$
\begin{aligned}
\sum_{\alpha=0}^{n}\left(\mathbf{R}_{m+1}^{(\alpha)}-\mathbf{R}_{m}^{(\alpha)}\right)^{2} & =\sum_{\beta=0}^{n}\left(\mathbf{X}_{m+1}^{(\beta)}-\mathbf{X}_{m}^{(\beta)}\right)^{2} \\
\sum_{\alpha=1}^{n}\left\{\mathbf{R}_{m}^{(\alpha)}-\boldsymbol{\lambda} \mathbf{R}_{m}^{(0)}\right\}^{2} & =\sum_{\beta=1}^{n} \mathbf{X}_{m+1}^{(\beta)}\left(\mathbf{1}+n \boldsymbol{\lambda}^{2}\right) .
\end{aligned}
$$

Note that the unperturbed Green function related to $\mathrm{e}^{-W}$ is that of the Brownian chain. In replicated coordinates this is $[8,12]$

$$
G_{0}\left(\left\{\mathbf{X}_{m}^{(\beta)}, \mathbf{X}_{m-1}^{(\beta)}\right\}\right)=\frac{1}{V \prod_{i}\left(1+n \lambda_{i}^{2}\right)^{1 / 2}}+\left(\frac{3}{2 \pi \ell \sigma}\right)^{3 / 2} \exp \left(-\frac{3}{2 \ell \sigma} \sum_{m}\left(\mathbf{X}_{m}^{(\beta)}-\mathbf{X}_{m-1}^{(\beta)}\right)^{2}\right)
$$

Note that $V$ is the volume of the $(0)$ system and $V \prod_{i}\left(1+n \lambda_{i}^{2}\right)^{1 / 2}$ that of the replicated system.

It might be useful to note that $\int \mathrm{e}^{-W-U}$ corresponds to the Green function of a harmonic oscillator [16], written in discrete coordinates. One can simplify the analysis drastically if only the limit of long rods is considered, and the ground state dominance of the Green function is used, i.e. symbolically written as

$$
\left(\frac{q_{0}}{\pi^{2}}\right)^{3 / 2} \exp \left(-\frac{1}{2} q_{0}\left\{\mathbf{X}_{1}^{2}+\mathbf{X}_{2}^{2}\right\}-\frac{1}{2} \ell \sigma\left(k_{1}-k_{2}\right)\right)
$$

The $k$ 's describe the arc-distance between the two points $\mathbf{X}_{1}, \mathbf{X}_{2}$.

The averages can now be calculated, and we give the results. First $\left(Q_{m}\right)$ :

$$
\begin{array}{r}
\left\langle Q_{m}\right\rangle=4\left(\frac{\alpha\left(q_{0}\right)}{2}\right)^{3 n / 2} \int \frac{\mathrm{d} \boldsymbol{\varepsilon}}{V \prod_{i}\left(1+n \lambda_{i}^{2}\right)^{1 / 2}} \exp \left(\sum_{i} \frac{2}{\alpha\left(q_{0}\right)}(n+1) \varepsilon_{i}^{2}\right) \frac{\sinh 2|\boldsymbol{\varepsilon}| \xi}{2|\boldsymbol{\varepsilon}| \xi} \times \\
\times\left(\exp \left\{-2(n+1) / \alpha\left(q_{0}\right)\left[\xi^{2}-\sum_{i} \lambda_{i}^{2} \varepsilon_{i}^{2}\right]\right\} \frac{\sinh 2|\boldsymbol{\varepsilon} \boldsymbol{\lambda}| \xi}{2|\boldsymbol{\varepsilon} \boldsymbol{\lambda}| \xi}\right) .
\end{array}
$$

The average for the trial potential is

$$
\langle U\rangle=1 / 4 \ell \sigma n q_{0}^{2} /\left(2 \alpha\left(q_{0}\right)\right)
$$


and finally for the crosslink term $\langle X\rangle$ we find

$$
\langle X\rangle=\prod_{i}\left(\frac{\alpha\left(q_{0}\right)}{2}\right)^{3 n / 2} \frac{\ell \sigma}{V \prod_{i}\left(1+n \lambda_{i}^{2}\right)^{1 / 2}}+\text { cut off terms }
$$

where we have note written the cut off terms because these are not part of the deformation dependency of the whole lot. Finally we note that the free energy can be calculated by $\left.\frac{\partial\left\langle Z^{n}\right\rangle}{\partial n}\right|_{n=0}$, leading to the result in the main text.

\section{Appendix B.}

THE SIMPLEST ALGEBRA OF THE SECOND QUANTIZATION METHOD USES EXPONENTIALS. - The exponent for three functional crosslinkage is :

$$
\begin{array}{r}
\varepsilon=-\int \Phi \Phi^{*}+v \int \Phi^{* 3}+\mu \iint \Phi g \Phi- \\
-M \log v-N \log \mu .
\end{array}
$$

Take as trials exponentials, i.e.

$$
\Phi^{*}=A \exp \left(-\alpha r^{2}\right), \quad \Phi=B \exp \left(-\beta r^{2}\right)
$$

we find form the integrals in $\varepsilon$, apart from uninteresting constants like $\pi$, etc. the following functions $a, b, c$ of the trials $\alpha$ and $\beta . g$ is taken to be exponential as well, containing $\xi$.

$$
\begin{aligned}
& \int \Phi^{* 3}=A^{3} a(\alpha) \\
& \int \Phi \Phi^{*}=A B c(\alpha+\beta) \\
& \iint \Phi g \Phi=B^{2} \exp \left(-\beta \xi^{2} / 2\right) \equiv B^{2} b(\beta) .
\end{aligned}
$$

Thus the exponant $\varepsilon$ can be written as

$$
\begin{aligned}
\varepsilon=v A^{3} a(\alpha)+\mu B^{2} b(\beta)-A B c- \\
-M \log v-N \log \mu .
\end{aligned}
$$

To find the minimum of this we differentiate with respect to the parameters $\alpha, \beta$ and find the system of differential equations

$$
\begin{gathered}
v A^{3} a^{\prime}-A B c^{\prime}=0 \\
\mu B^{2} b^{\prime}-A B c^{\prime}=0 \\
3 v A^{2} a-B c=0 \\
2 \mu B b-A c=0 \\
v A^{3}=N, \quad \mu B^{2} b=M .
\end{gathered}
$$

The dash denotes derivatives with respect to the appropriate variables. These give the relations

$$
\begin{aligned}
& 1 / 3 a \frac{\partial a}{\partial \alpha}=1 / c \frac{\partial c}{\partial \alpha} \\
& 1 / 2 b \frac{\partial b}{\partial \beta}=1 / c \frac{\partial c}{\partial \beta}
\end{aligned}
$$

from which it is easily obtained that

$$
\begin{aligned}
& \beta=2 \alpha \\
& \beta=1 /\left(2 \xi^{2}\right)
\end{aligned}
$$

as quoted in the main text.

The evaluation of the free energy using this exponential trials is now the same as mentioned in (3.5), i.e. integrals such as

$$
\begin{aligned}
\int \mathrm{d}^{3} R \int & \exp \left\{-\frac{\beta}{2}(\mathbf{R}-\boldsymbol{\varepsilon} \xi)\right\} \mathrm{d} \boldsymbol{\varepsilon} \times \\
& \times \log \left\{\int \exp \left\{-\frac{\beta}{2}(\boldsymbol{\lambda R}-\boldsymbol{\varepsilon} \xi)\right\} \mathrm{d} \boldsymbol{\varepsilon}\right\}
\end{aligned}
$$

which is again not simple. Alternatively one can employ the integral equation and try to make assumptions and simplify the propagator $g$. All these feasible approaches give always the classical $\lambda^{2}$ structure, as in all classical theories. This is clearly due to the use of the best Gaussian, but in the rod network this seems to be only marginally adequate.

\section{References}

Paper I is the following paper: Edwards S. F. 49 (1988) 1673.

[1] Aharony, S., private communication.

[2] WeGNer, G., private communication.

[3] Maxwell, J. C., Trans. R. Soc. Edinburgh 26 (1970) 1.

[4] Wall, F. T. and Flory, P. J., J. Chem. Phys. 19 (1951) 1435.
[5] James, H. M. and Guth, E., J. Chem. Phys. 11 (1943) 455.

[6] BAStide, J., Physics of Finely Divided Matter, Eds. N. Boccara and M. Daoud (Springer, Heidelberg) 1985.

[7] BAstide, J. and Boué, F., Physica 140A (1986) 251 ; Boué, F. and Bastide, J., Prog. Coll. Polym. Sci. (1987) to app. 
[8] Warner, M. and Edwards, S. F., J. Phys. A 11 (1978) 1649.

[9] Vilgis, T. A. and Boué, F., Polymer 27 (1986) 1154.

[10] Stein, R. S., Phys. Today 78 (1981) 482.

[11] Edwards, S. F., Polymer Networks Eds. A. J. Chrompff and S. Newman (Plenum Press, New York) 1971.

[12] Deam, R. T. and Edwards, S. F., Phil. Trans. $R$. Soc. A 280 (1976) 317.

[13] Edwards, S. F. and Anderson, P. W., J. Phys. F 5 (1975) 965.

[14] Edwards, S. F. and Vilgis, T. A., Rep. Prog. Phys. 51 (1988) 243.

[15] Vilgis, T. A., Comprehensive Polym. Sci., Ed. G. C. Eastmond (Pergamon Press, Oxford) Vol. 5.
[16] Feynman, R. P. and Hibbs, A. R., Pathintegrals and Quantum Mechanics (Mc Graw Hill, New York) 1965.

[17] Edwards, S. F. and Vilgis, T. A., Polymer 27 (1986) 483.

[18] DoI, M. and Edwards, S. F., Theory of Polymer Dynamics (Oxford University Press, Oxford) 1986.

[19] Edwards, S. F. and Vilgis, T. A., Physica Scripta T 13 (1986) 7.

[20] Edwards, S. F., Proc. Phys. Soc. 88 (1966) 265 ; Edwards, S. F., Proc. Phys. Soc. 91 (1967) 513.

[21] Vilgis, T. A. and Boué, F., J. Pol. Sci. (1988) in press. 